\section{Hepatitis B Virus Screening and Potential Reactivation in Patients Undergoing Treatment for Cancer}

Sarah P. Hammond, MD; Sankar Swaminathan, MD; William I. Bensinger, MD; and Lindsey R. Baden, MD

An estimated 2 billion people worldwide have been infected with hepatitis $B$ virus (HBV). Of those, an estimated 240 million have chronic HBV infection. ${ }^{1,2}$ In the United States, where HBV is nonendemic, an estimated 1.4 million people have chronic HBV infection, and more have experienced a previous infection that has "resolved." $\mathrm{HBV}$ not only is linked to an increased risk for developing hepatocellular carcinoma but also confers risk of HBV-associated morbidity in patients with chronic or previous HBV infection undergoing immunosuppressive therapy, such as with certain types of monoclonal antibodies or cancer treatment.

HBV can reactivate in the context of cancer chemotherapy treatment both in patients with chronic HBV infection (detectable hepatitis B surface antigen [HBsAg] in serum) and in those with resolved HBV infection (detectable hepatitis B core immunoglobulin $\mathrm{G}[\mathrm{IgG}]$ antibody $[\mathrm{HBcAb}]$ and negative $\mathrm{HBsAg}$ ). Studies examining HBV reactivation typically define it as redevelopment of active HBV replication with at least a 10-fold increase in detectable HBV DNA. Generally, this increase precedes or is concurrent with the development of active liver inflammation and redevelopment of HBsAg in those with previous evidence of resolved HBV infection. The clinical illness associated with $\mathrm{HBV}$ reactivation can progress from asymptomatic elevations of liver enzymes to hepatic synthetic dysfunction, hepatic failure, and death. In addition, HBV reactivation during cancer chemotherapy has been linked to an increased risk of chemotherapy delay and premature termination of chemotherapy, both of which may lead to poorer cancer outcomes. ${ }^{4}$ Most studies examining hepatitis in the setting of cancer chemotherapy have been performed in Asia and other countries where HBV infection is endemic, but multiple large cancer centers in the United States have also studied this issue. ${ }^{5-7}$ With the advent of improved diagnostic tools (ie, quantitative HBV DNA measurement) and the development of active HBV antiviral therapies (eg, lamivudine, tenofovir, entecavir), the potential to more carefully monitor and treat HBV makes the identification of those at risk for HBV reactivation an important issue to address.

The risk for HBV reactivation during chemotherapy varies depending on both the patients' hepatitis B status and the chemotherapeutic agent used. In general, the reactivation risk is higher among individuals with chronic HBV infection ( $\mathrm{HBs} A g$ positive) than among those with evidence of resolved infection (HBsAg-negative, $\mathrm{HBcAb}$-positive). Based on cancer therapy type, reactivation risk appears to be highest in patients receiving monoclonal therapy directed at CD20 and hematopoietic stem cell transplant (HSCT) recipients. Both rituximab and ofatumumab have black box warnings about the risk for HBV reactivation leading to liver failure and death. Thus, screening for HBV before treatment is recommended. The rate of reactivation among patients treated with rituximab-based chemotherapy for nonHodgkin's lymphoma ranges from $16 \%$ to $24 \%$ in those with resolved HBV infection, but reached $80 \%$ in one study of patients with chronic HBV infection. ${ }^{8-10}$ Other specific systemic cancer and immunosuppressive therapies that have been linked to HBV reactivation include alemtuzumab, temozolamide, imatinib, anthracyclines, and corticosteroids. ${ }^{11-13}$ In addition, HBV reactivation has been reported in patients with hepatocellular carcinoma treated with transarterial chemoembolization without

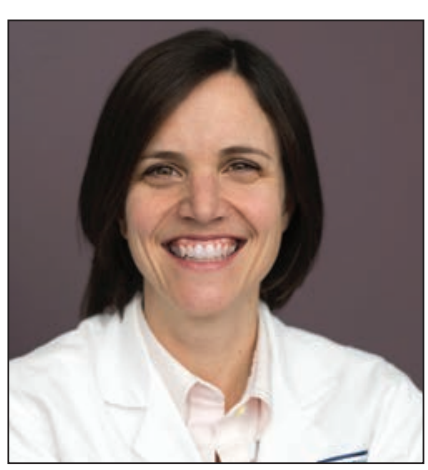

Sarah P. Hammond, MD

Sarah Hammond, MD, is Instructor in Medicine at Harvard Medical School, is on the clinical faculty in the Infectious Diseases Division at Brigham and Women's Hospital, and provides consultative services at Dana-Farber Cancer Institute. Her clinical research interests include the improvement of infectious diseases care for cancer and transplant patients, with a focus on the prevention and treatment of hepatitis B reactivation.

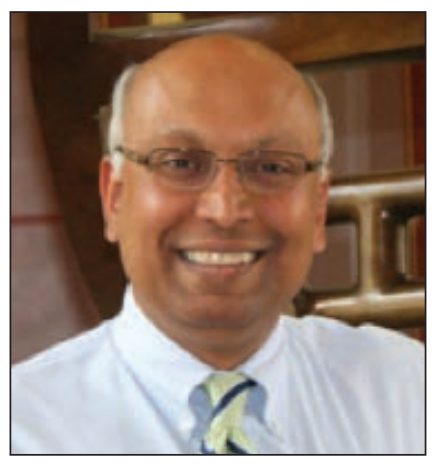

Sankar Swaminathan, MD

Sankar Swaminathan, MD, is Chief of Infectious Diseases at the University of Utah. In this role he emphasizes the importance of both basic-science and clinical research priorities. Dr. Swaminathan's research focuses on the regulation of gene expression in Epstein-Barr virus and Kaposi sarcoma-associated herpes virus.

The ideas and viewpoints expressed in this editorial are those of the author and do not necessarily represent any policy, position, or program of NCCN. 


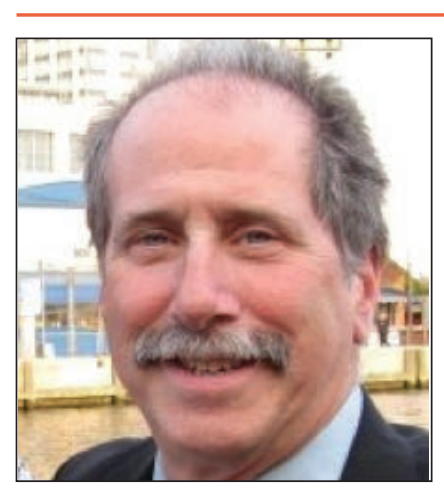

William I. Bensinger, MD

William Bensinger, MD, is Director of the Autologous Stem Cell Transplant Program at Fred Hutchinson Cancer Research Center. He is a full member at the Center, and Professor of Medicine at the University of Washington. He is Vice-Chair of the NCCN Guidelines Panel for Multiple Myeloma and a Co-Chair of the NCCN Guidelines Panel for Prevention and Treatment of Cancer-Related Infections. He is a member of the International Myeloma Working Group clinical endpoints committee.

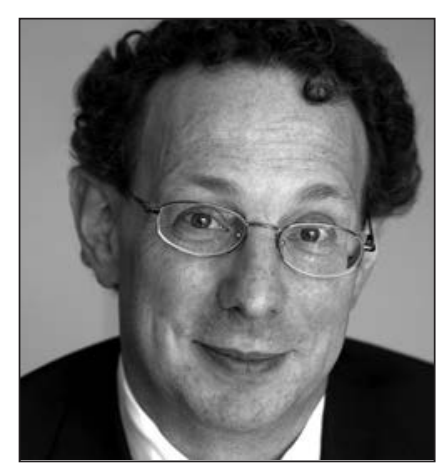

Lindsey R. Baden, MD

Lindsey Baden, MD, is Director of Infectious Diseases at DanaFarber Cancer Institute and an Associate Professor of Medicine at Harvard Medical School. He is an active investigator, clinician, and educator. He Co-Chairs the NCCN Guidelines Panel for Prevention and Treatment of Cancer-Related Infections. systemic chemotherapy. ${ }^{14}$ Multiple small studies and a meta-analysis have shown that prophylactic antiviral therapy in high-risk patients can prevent reactivation and associated hepatic morbidity, and also reduce the risk of chemotherapy interruption. ${ }^{15,16}$

An issue of debate is whether to screen patients with cancer for chronic and resolved HBV infection before administering/initiating therapies other than HSCT or treatment directed at CD20 in order to identify patients at risk for reactivation. Unfortunately, limited data are available examining the cost-effectiveness and clinical utility of this screening. Nonetheless, several national guidelines, including the NCCN Clinical Practice Guidelines in Oncology (NCCN Guidelines) for the Prevention and Treatment of Cancer-Related Infections provide expert guidance on the topic.

The US Preventative Services Task Force recently updated their guidelines for HBV screening in the general population. These guidelines indicate that screening should be performed not only in patients infected with HIV, those who use intravenous drugs, and men who have sex with men, but also in all patients born in a country where the prevalence of $\mathrm{HBV}$ is greater than $2 \%$ or in those born to parents from such a country and were not vaccinated for HBV during infancy. ${ }^{17}$ In line with these recommendations and those of ASCO and the American Association for the Study of Liver Diseases, NCCN Guidelines also suggest risk-based screening of all patients with cancer. ${ }^{18-20}$

However, for busy practicing oncologists, the feasibility of assessing risk factors associated with HBV infection in patients who will be treated with chemotherapy is not clear. Furthermore, among more than 1000 patients with recently diagnosed acute HBV infection reported to the Centers for Disease Control and Prevention, more than half had no identifiable HBV risk factors or exposures. ${ }^{21}$ In recognizing the nuanced history-taking required to appropriately screen patients with cancer for HBV risk and the potential insensitivity of such histories to identify those at risk, NCCN Guidelines indicate that universal screening with $\mathrm{HBs} A g$ and $\mathrm{HBcAb}$ should be considered for centers where risk-based screening cannot be effectively performed. Indeed, some large cancer centers have recently moved to universal screening for HBV infection in an effort to simplify screening practices, improve screening compliance, and ultimately prevent HBV reactivation-related morbidity and mortality through early identification and intervention for patients determined to be at risk. ${ }^{22}$

HBV reactivation, although uncommon in patients with cancer who are receiving therapies other than anti-CD20 agents and HSCT is an increasingly recognized preventable complication of cancer therapy associated with significant potential morbidity and mortality. Further studies of the general population of patients with cancer, with a focus on those with solid tumor malignancies, are needed to delineate optimal screening practices and management of patients with chronic and resolved HBV infection. Until the results of such studies are available, each cancer center, practice, and individual provider must carefully consider the practicability of riskbased versus universal screening for HBV.

\section{References}

1. Hepatitis B vaccines. Wkly Epidemiol Rec 2009;84:405-419.

2. Hepatitis B. World Health Organization Web site. Available at: http://www.who.int/mediacentre/factsheets/fs204/ en/. Accessed September 18, 2014.

3. Centers for Disease Control and Prevention. Viral hepatitis statistics and surveillance. Available at: http://www.cdc. gov/hepatitis/Statistics/index.htm. Accessed September 18, 2014.

4. Yeo W, Chan PK, Hui $\mathrm{P}$, et al. Hepatitis $\mathrm{B}$ virus reactivation in breast cancer patients receiving cytotoxic chemotherapy: a prospective study. J Med Virol 2003;70:553-561.

5. Hammond SP, Borchelt AM, Ukomadu C, et al. Hepatitis B virus reactivation following allogeneic hematopoietic stem cell transplantation. Biol Blood Marrow Transplant 2009;15:1049-1059. 
Hepatitis B Screening in Patients With Cancer

6. Ramos CA, Saliba RM, de Padua Silva L, et al. Resolved hepatitis B virus infection is not associated with worse outcome after allogeneic hematopoietic stem cell transplantation. Biol Blood Marrow Transplant 2010;16:686-694.

7. Reed EC, Myerson D, Corey L, Meyers JD. Allogeneic marrow transplantation in patients positive for hepatitis B surface antigen. Blood 1991;77:195-200.

8. Yeo W, Chan TC, Leung NW, et al. Hepatitis B virus reactivation in lymphoma patients with prior resolved hepatitis B undergoing anticancer therapy with or without rituximab. J Clin Oncol 2009;27:605-611.

9. Niitsu N, Hagiwara Y, Tanae K, et al. Prospective analysis of hepatitis B virus reactivation in patients with diffuse large B-cell lymphoma after rituximab combination chemotherapy. J Clin Oncol 2010;28:5097-5100.

10. Pei SN, Chen $\mathrm{CH}$, Lee $\mathrm{CM}$, et al. Reactivation of hepatitis $\mathrm{B}$ virus following rituximab-based regimens: a serious complication in both HBsAg-positive and HBsAg-negative patients. Ann Hematol 2010;89:255-262.

11. Iannitto E, Minardi V, Calvaruso G, et al. Hepatitis B virus reactivation and alemtuzumab therapy. Eur J Haematol 2005;74:254-258

12. Hwang JP, Vierling JM, Zelenetz AD, et al. Hepatitis B virus management to prevent reactivation after chemotherapy: a review. Support Care Cancer 2012;20:2999-3008.

13. Kang BW, Lee SJ, Moon JH, et al. Chronic myeloid leukemia patient manifesting fatal hepatitis $B$ virus reactivation during treatment with imatinib rescued by liver transplantation: case report and literature review. Int J Hematol 2009;90:383-387.

14. Peng JW, Lin GN, Xiao JJ, Jiang XM. Hepatitis B virus reactivation in hepatocellular carcinoma patients undergoing transcatheter arterial chemoembolization therapy. Asia Pac J Clin Oncol 2012;8:356-361.

15. Yeo W, Chan PK, Ho WM, et al. Lamivudine for the prevention of hepatitis B virus reactivation in hepatitis $B$ s-antigen seropositive cancer patients undergoing cytotoxic chemotherapy. J Clin Oncol 2004;22:927-934.

16. Loomba R, Rowley A, Wesley R, et al. Systematic review: the effect of preventive lamivudine on hepatitis B reactivation during chemotherapy. Ann Intern Med 2008;148:519-528.

17. LeFevre ML. Screening for hepatitis B virus infection in nonpregnant adolescents and adults: U.S. Preventive Services Task Force recommendation statement. Ann Intern Med 2014;161:58-66.

18. Artz AS, Somerfield MR, Feld JJ, et al. American Society of Clinical Oncology provisional clinical opinion: chronic hepatitis B virus infection screening in patients receiving cytotoxic chemotherapy for treatment of malignant diseases. J Clin Oncol 2010;28:3199-3202.

19. Lok AS, McMahon BJ. Chronic hepatitis B: update 2009. Hepatology 2009;50:661-662.

20. Baden LR, Bensinger W, Angarone M, et al. NCCN Clinical Practice Guidelines in Oncology: Prevention and Treatment of Cancer-Related Infections. J Natl Compr Canc Netw 2012;10:1412-1445.

21. Centers for Disease Control and Prevention. Surveillance for viral hepatitits-United States, 2012. Available at: http://www.cdc.gov/hepatitis/Statistics/2012Surveillance/index.htm. Accessed September 19, 2014.

22. Sammons MK, Patel D, Sepkowitz EJ, et al. Successful implementation of a universal screening program to prevent hepatitis B reactivation in a large cancer center. Hepatology 2013;58:607A-626A. 